\title{
In-vitro mechanical behavior and in-vivo healing response of a new generation biodegradable polymer-coated thin-strut sirolimus-eluting stents
}

\author{
Paweł Gąsior ${ }^{1}$, Łukasz Pyka², Michał Hawranek², Michał Garbacz ${ }^{3}$, Madeline Chagnonn ${ }^{4}$, Diane Beaudry4, \\ Joanna Fluder', Mariusz Gąsior², Wojciech Wojakowski', Andrzej Lekston² \\ 'Department of Cardiology and Structural Heart Diseases, Medical University of Silesia, Katowice, Poland \\ ${ }^{2} 3^{\text {rd }}$ Department of Cardiology, School of Medicine with the Division of Dentistry in Zabrze, Medical University of Silesia, Katowice, Silesian Center for Heart Diseases, \\ Zabrze, Poland \\ ${ }^{3}$ Department of Cardiac, Vascular and Endovascular Surgery and Transplantology, Silesian Center for Heart Diseases in Zabrze, Zabrze, Poland \\ ${ }^{4}$ Charles River Laboratories, Montreal, Canada
}

Correspondence to:

Paweł Gąsior, MD, PhD

Division of Cardiology and

Structural Heart Diseases,

Medical University of Silesia,

Ziołowa 47, 40-635 Katowice,

Poland,

phone: +48 322523930 ,

e-mail: p.m.gasior@gmail.com

Copyright by the Author(s), 2022

DOI: 10.33963/KP.a2021.0158

Received:

May 27, 2021

Accepted:

November 5, 2021

Early publication date:

November 5, 202

\section{INTRODUCTION}

First-generation drug-eluting stents (DES) coated with durable polymers reduced rates of restenosis when compared with bare-metal stents. Although DES achieved great results in inhibiting neointimal hyperplasia, the presence of durable polymers was associated with the added risks of stent thrombosis (ST) [1, 2]. The introduction of second-generation DES resulted in reduced rates of ST with preserved low restenosis rates [3]. However, very late ST and neo-atherosclerosis have been recently observed with second-generation DES $[2,4]$. Biodegradable polymers DES were designed to reduce local inflammatory and hypersensitivity reactions responsible for delayed arterial healing, which is the primary substrate underlying late ST [5]. Contemporary DES are increasingly used for the treatment of complex lesions such as left main artery and long bifurcated segments. Moreover, stent post-dilatation is frequently performed, typically with large over-expansion in the setting of the long tapering vessel segment. Therefore, the information regarding the overexpansion capabilities of each device is crucial in clinical practice.

In this study, we aim to evaluate in-vitro mechanical behavior and in-vivo healing response of a new-generation biodegradable polymer-coated thin-strut sirolimus-eluting stent (Alex Stratos, Balton, Warszawa, Poland) in comparison with the commercially available previous generation platform (Alex Plus Bal- ton, Warszawa, Poland) and the leading DES (Orsiro, Biotronik, Bülach, Switzerland).

\section{METHODS}

\section{Device description}

Alex Stratos is a CE mark approved new generation biodegradable polymer-coated sirolimus-eluting stent with a laser-cut cobalt-chromium alloy platform with a wall thickness of $70 \mu \mathrm{m}$. It has an improved design with open cells for unobstructed side branch access (Figure 1A). Unlike other contemporary DES which typically has two or three designs to cover the entire range of their diameter, Alex Stratos has an individual design every $0.5 \mathrm{~mm}$ in diameter, which allows more uniform drug distribution (data on file at Balton). The device is covered with a multilayer structure containing a poly-L-lactide polymer and sirolimus in a dose of $1.2-1.4 \mathrm{mg} / \mathrm{mm}^{2}$. The proprietary polymer technology allows maintaining polymer integrity during aggressive overexpansion of the stent up to $1.5 \mathrm{~mm}$ above nominal diameter (Figure 1B). In this study, Alex Stratos was compared in an in-vitro and in-vivo setting with the commercially available previous generation platform Alex Plus whose platform is made of a cobalt-chromium alloy with a wall thickness of $70 \mu \mathrm{m}$. Alex Plus is covered with the same multilayer structure as Alex Stratos. Both Alex Stratos and Alex Plus were evaluated in the in-vitro setting against 


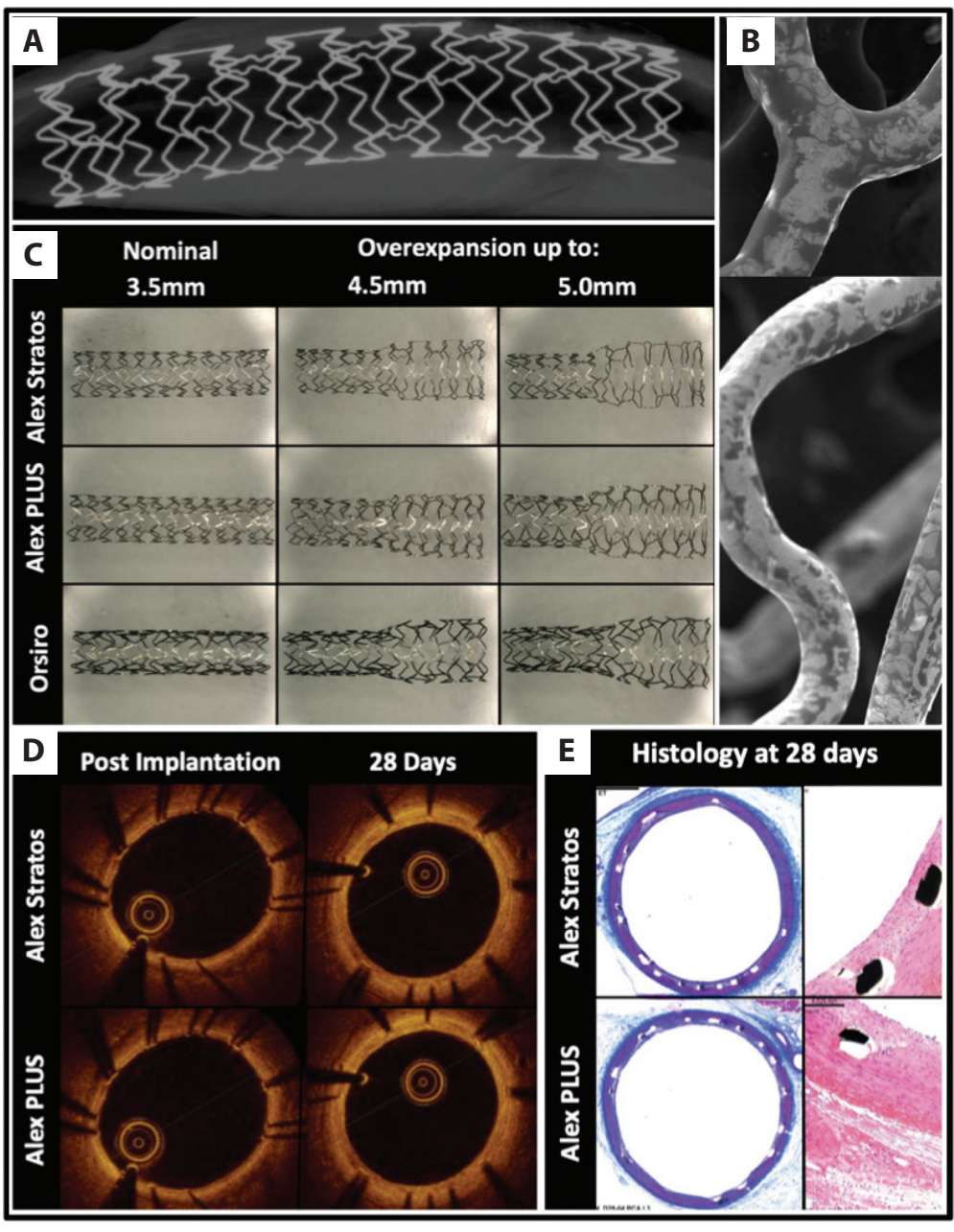

Figure 1. A. High-resolution radiography image of Alex Stratos new design with open cells. B. Polymer coating integrity after post-dilatation $1.5 \mathrm{~mm}$ above nominal stent diameter. C. Light digital microscopy images of Alex Stratos, Alex Plus, and Orsiro deployed at a nominal diameter $(3.5 \mathrm{~mm})$ and post-dilatated up to $4.5 \mathrm{~mm}$ and $5.0 \mathrm{~mm}$. D. Representative optical coherence tomography images at the $28^{\text {th }}$ day follow-up. E. Representative histology images at 2-day follow-up leading cobalt-chromium biodegradable polymer-coated sirolimus-eluting stent Orsiro.

\section{In-vitro overexpansion testing}

The mechanical behavior of the investigated Alex Stratos and commercially available Alex Plus and Orsiro stents, following over-expansion, was examined under static conditions. All tested devices were $3.5 \times 15 \mathrm{~mm}$ and were deployed in-vitro at nominal pressure. Subsequently, over-expansion results for each design were tested with successive post-dilations using $4.5 \times 12 \mathrm{~mm}$ and semi-compliant balloons inflated at $8 \mathrm{~atm}$ followed by a $5.0 \times 12 \mathrm{~mm}$ non-compliant balloon with a pressure of $18 \mathrm{~atm}$. Post-dilation was performed on the proximal segment. Final dilations were repeated on the samples a second time to ensure an optimal expansion of the stent struts. For each stent, two samples were deployed. Stent samples were mounted and analyzed using light digital microscopy and evaluated using the previously described methodology (Figure 1C) [6].

\section{In-vivo healing study}

The study protocol was approved by the Local Ethics Committee for animal research. All animals received a standard of care outlined in the study protocol and in accordance with the act of animal welfare and the "Principles of Care of Laboratory Animals" [7]. In this study, either Alex Stratos $(n=11)$ or Alex Plus $(n=24)$ were implanted targeting a stent-to-artery ratio of 1.1:1 under online quantitative coronary angiography (QCA) guidance in 35 coronary arteries of 12 pigs. Control coronary angiography, optical coherence tomography (OCT), and a histological evaluation were performed 28 days following stent implantation. QCA analysis was performed using QAngio XA Software ${ }^{\mathrm{TM}}$ 7.1.14.0 (Medis Medical Imaging System, Leiden, Netherlands). OCT images were recorded using the ILUMIEN PCI Optimization System (St. Jude Medical, St. Paul, MN, USA), and the qualitative analyses were performed with the commercial software (ILUMIEN OPTIS, St. Jude Medical, St. Paul, MN, USA). An independent pathology laboratory (AccelLAB, Boisbriand, Canada) conducted the histological evaluation.

\section{Statistical analysis}

Statistical analysis was performed using SigmaStat statistical software (version 13.1; Systat Software, San Jose, CA, USA). The normality of the distribution was verified using the Shapiro-Wilk test. Due to skewed distributions, the median (interquartile range) was provided. QCA, OCT, 
and histological data were analyzed with a nonparametric Mann-Whitney test. Differences were only considered significant when the calculated $P$-value was $<0.05$. In-vitro overexpansion evaluation was performed for exploratory purposes and due to the small sample size, statistical analysis was not performed.

\section{RESULTS AND DISCUSSION}

In-vitro overexpansion testing in static conditions demonstrated that all tested stents were able to expand well above their labeled maximal stent diameter using larger post-dilatation balloons. From the tested devices, only Alex Plus presented signs of strut fracture $(n=2)$ when over-expanded at $1.5 \mathrm{~mm}$ above nominal diameter (42\% overexpansion). This indicates improvement in the Alex Stratos architecture when compared to the previous generation Alex Plus. Stents are rarely able to achieve a minimal lumen diameter equal to the diameter of the balloon used for post-dilatation [8]. In the presented study, Alex Stratos achieved a minimal lumen diameter closest to the expected balloon diameter during post-dilatation using a 5.0 non-compliant balloon (Alex Stratos $4.98 \mathrm{~mm}$ and $5.01 \mathrm{~mm}$ vs. Alex Plus $4.82 \mathrm{~mm}$ and $4.86 \mathrm{~mm}$ vs. Orsiro $4.76 \mathrm{~mm}$ and $4.93 \mathrm{~mm}$ ). The overexpansion capabilities are especially important in the bifurcation setting where a significant discrepancy between the distal and proximal diameter is present. Cell opening at nominal diameter appeared to be numerically largest in Alex Stratos when compared to Alex Plus and Orsiro $\left(1.32 \mathrm{~mm}^{2}\right.$ and $1.34 \mathrm{~mm}^{2}$ vs. $1.09 \mathrm{~mm}^{2}$ and $1.13 \mathrm{~mm}^{2}$ vs. $1.28 \mathrm{~mm}^{2}$ and $1.36 \mathrm{~mm}^{2}$, respectively) and increased by $125 \%$ and $127 \%$ in Alex Stratos, $112 \%$ and $120 \%$ in Alex Plus, and $126 \%$ and $130 \%$ in Orsiro between nominal pressure deployment and maximal overexpansion. Side branch access is very important especially during the treatment of complex lesions such as bifurcations. The improved design of Alex Stratos allowed an increase in mean cell opening by almost $10 \%$ when compared to the previous generation Alex Plus achieving similar results to the Orsiro stent. Therefore, due to enhanced overexpansion capabilities and greater cell opening Alex Stratos might be better suited for bifurcation interventions including lesions requiring a two-stent approach when compared to the previous generation Alex Plus. However, due to the small sample size and experimental nature of this study, these results must be interpreted with caution.

The in-vivo healing response study evaluated the vascular response to Alex Stratos when compared to Alex Plus. The appropriateness of applied methodology was confirmed by comparable balloon-to-artery ratio, vessel, and stents sizes during the implantation procedure evaluated by QCA. At 1 month, both devices demonstrated comparable late lumen loss (Alex Stratos $=0.13$ [0.00-0.40] $\mathrm{mm}$ vs. Alex Plus $=0.10[0.05-0.28] \mathrm{mm} ; P=0.54)$. OCT analysis demonstrated that there was no difference in the stent area between Alex Stratos and Alex Plus (8.41 [6.26-9.32] $\mathrm{mm}^{2}$ vs. 7.40 [5.97-8.99] $\mathrm{mm}^{2}$ respectively; $\left.P=0.674\right]$ at the $28^{\text {th }}$ day follow-up. Furthermore, the percentage areas of stenosis (\%AS: Alex Stratos 9.62 [7.20-12.93] \% vs. Alex Plus 10.47 [9.26-12.67] \%; $P=0.899$ ) were similar between the tested stents (Figure 1E). Histological assessment revealed that vascular responses to Alex Stratos were comparable with those to Alex Plus at day 28 of follow-up. Morphometric analysis showed no difference in the internal elastic lamina or the external elastic lamina areas between both devices. Percent area stenosis (\%AS) were numerically smaller in Alex Stratos when compared to Alex Plus (12.1 [9.7-13.3] \% vs. 15.2 [8.9-22.8]\% respectively; $P=0.317]$ ) (Figure 1E). There was no difference in inflammation scores between the tested groups. Fibrin score was numerically higher in the Alex Stratos group, but it remained at a low level, and the difference did not reach statistical significance (Alex Stratos 0.52 [0.33-0.81] vs. Alex Plus 0.22 [0.15-0.46], $P=0.055$ ). Furthermore, fibrin score in both tested groups was numerically lower than in the previously reported preclinical data for other sirolimus, as well as everolimus and zotarolimus-eluting stents $[9,10]$. Finally, the animal model of in-stent restenosis in healthy coronary arteries used in this study cannot reproduce the complexity of coronary artery disease in humans, therefore, the results have to be interpreted with caution.

In summary, the new-generation Alex Stratos demonstrated improved biomechanical behavior to the previous generation platform (Alex Plus) with results similar to the Orsiro stent. Furthermore, Alex Stratos demonstrated a favorable healing profile in the in-vivo setting. Our findings suggest that the new-generation Alex Stratos tested in this study has the potential to improve the performance shown by the current generation Alex Plus by providing a highly biocompatible platform with significantly improved mechanical properties.

\section{Article information}

Funding: The study was funded by Balton, Warszawa, Poland. Conflict of interest: None declared.

Open access: This article is available in open access under Creative Common Attribution-Non-Commercial-No Derivatives 4.0 International (CC BY-NC-ND 4.0) license, allowing to download articles and share them with others as long as they credit the authors and the publisher, but without permission to change them in any way or use them commercially. For commercial use, please contact the journal office at kardiologiapolska@ptkardio.pl.

\section{REFERENCES}

1. Nebeker JR, Virmani R, Bennett CL, et al. Hypersensitivity cases associated with drug-eluting coronary stents: a review of available cases from the Research on Adverse Drug Events and Reports (RADAR) project. J Am Coll Cardiol. 2006; 47(1): 175-181, doi: 10.1016/j.jacc.2005.07.071, indexed in Pubmed: 16386683.

2. Park SJ, Kang SJ, Virmani R, et al. In-stent neoatherosclerosis: a final common pathway of late stent failure. J Am Coll Cardiol. 2012; 59(23): 20512057, doi: 10.1016/j.jacc.2011.10.909, indexed in Pubmed: 22651862.

3. Dores H, Raposo L, Campante Teles R, et al. Stent thrombosis with secondversus first-generation drug-eluting stents in real-world percutaneous coronary intervention: analysis of 3806 consecutive procedures from 
a large-volume single-center prospective registry. J Invasive Cardiol. 2013; 25(7): 330-336, indexed in Pubmed: 23813060.

4. Nakazawa G, Otsuka F, Nakano M, et al. The pathology of neoatherosclerosis in human coronary implants bare-metal and drug-eluting stents. J Am Coll Cardiol.2011;57(11):1314-1322, doi: 10.1016/j.jacc.2011.01.011, indexed in Pubmed: 21376502.

5. Joner $M, F i n n A V$, Farb A, et al. Pathology of drug-eluting stents in humans: delayed healing and late thrombotic risk. J Am Coll Cardiol. 2006; 48(1): 193-202, doi: 10.1016/j.jacc.2006.03.042, indexed in Pubmed: 16814667.

6. $\mathrm{Ng}$ J, Foin N, Ang HY, et al. Over-expansion capacity and stent design model: An update with contemporary DES platforms. Int J Cardiol. 2016; 221: 171-179, doi: 10.1016/j.jicard.2016.06.097, indexed in Pubmed: 27400317.

7. Institute of Laboratory Animal Resources NRC. Principles of Care of Laboratory Animals. NIH Publication. No. 85-23 1996.
8. Foin N, Sen S, Allegria E, et al. Maximal expansion capacity with current DES platforms: a critical factor for stent selection in the treatment of left main bifurcations? Eurolntervention. 2013; 8(11): 1315-1325, doi: 10.4244/EIJV8111A200, indexed in Pubmed: 23086760.

9. Sperling C, Waliszewski MW, Kherad B, et al. Comparative preclinical evaluation of a polymer-free sirolimus-eluting stent in porcine coronary arteries. Ther Adv Cardiovasc Dis. 2019; 13: 1753944719826335, doi: 10.1177/1753944719826335, indexed in Pubmed: 30803407.

10. Lim KS, Jeong $\mathrm{MHo}$, Bae InHo, et al. Histopathological comparison among biolimus, zotarolimus and everolimus-eluting stents in porcine coronary restenosis model. Korean Circ J. 2013; 43(11): 744-751, doi: 10.4070/kcj.2013.43.11.744, indexed in Pubmed: 24363750. 